\section{Crystal-clear dynamics}

Most enzymes fluctuate between different conformations to accomplish their function, and catalytic or regulatory mechanisms often involve long-range motions. Although X-ray crystallography offers high-resolution views of proteins in different conformations, single or even multiple structures may not provide detailed information on the precise nature of allosteric communication between distant sites. To harvest functional dynamics information from X-ray data, van den Bedem, Fraser and colleagues have now developed a new algorithm called 'contact networks through alternate conformation transitions' (CONTACT). To identify interaction networks, qFit-an algorithm previously developed to extract conformational heterogeneity from X-ray diffraction datais first used to define possible alternative conformations. CONTACT then uses this information to calculate the van der Waals interactions across all alternative conformations to define those likely to propagate to other residues. As a test of CONTACT's ability to identify contact networks from experimental X-ray data, the authors looked at two enzymes known to undergo pronounced conformational exchanges during catalysis: cyclophilin A (CYPA) and dihydrofolate reductase (DHFR). CONTACT was able to identify multiple plausible transition pathways to known end states of CYPA, results in line with those from recent NMR studies. To study long-range perturbation in DHFR, the authors obtained high-resolution X-ray diffraction data sets of wildtype DHFR at both cryogenic and room temperatures. They identified a contact network that connects the dynamic FG loop, the NADP-binding pocket and the adenosine-binding domain of DHFR. CONTACT predicted that removal of NADP would disrupt coupling between the FG loop and the adenosine-binding domain, and this prediction was experimentally confirmed by NMR analysis of DHFR containing the G121V mutation, located within the FG loop. Similar analyses of another catalytically defective DHFR mutant unexpectedly revealed an expanded contact network resulting in nonproductive motion around the active site and loss of catalytic efficiency. CONTACT should be a useful tool to complement the current arsenal of methods aimed at deciphering protein conformational dynamics. (Nat. Methods doi:10.1038/nmeth.2592, published online 4 August 2013)

SL

\section{Chromatin affects copy gain}

Cancer cells are often characterized by copy-number alterations at specific regions of the genome, but little is known about how localized copy gain is achieved. Whetstine, Getz and colleagues now report that overexpression of the histone demethylase KDM4A in two different cell lines induces transient, localized copy gain without global chromosome instability. Analysis of The Cancer Genome Atlas data showed that KDM4A is amplified and overexpressed in certain cancers and correlates with poor outcome in ovarian cancer. Importantly, KDM4A-amplified tumors had increased copy gains for the same regions identified in cell culture. KDM4A-dependent copy gain was shown to require histone demethylase activity, and interference with H3K9 or H3K36 methylation promoted copy gain, whereas HP1 $\gamma$ overexpression antagonized it. KDM4A-dependent copy gain was

Written by Arianne Heinrichs, Stéphane Larochelle \& Beth Moorefield induced in less than 24 hours and required S phase. These copy gains were not stably inherited and were cleared by late $\mathrm{G} 2$ by yet-unknown mechanisms. However, as these regions contain putative oncogenes, the transient copy gain could potentially promote tumorigenesis while masking the originating event. To gain insight into how KDM4A is involved in generating copy gain, the authors identified KDM4Ainteracting proteins by mass spectrometry analysis and observed a significant enrichment for proteins involved in rereplication, including MCMs and DNA polymerases. Indeed, although KDM4A did not affect widespread rereplication, it did promote rereplication of a specific locus that exhibits copy gain. These observations were further supported by chromatin immunoprecipitation experiments indicating that KDM4A overexpression induces chromatin changes and recruitment of the replication machinery at this region. Although these studies raise many important questions, they begin to establish how copy-number changes could originate during tumorigenesis and the role of chromatin modifiers in this process. (Cell 154, 541-555, 2013)

$A H$

\section{Transition control}

Whereas early stages of embryogenesis are driven by maternal gene products, transcription of the zygotic genome is required to direct later events. Initiation of the embryo's transcription program occurs just before gastrulation at the midblastula transition (MBT) and is accompanied by changes in cellcycle length, checkpoint activation and the onset of cell motility required for morphogenesis. It has long been thought that these events are triggered when the embryo attains a threshold nuclear/cytoplasmic (N/C) ratio, and an unknown, titratable factor becomes limiting through its interactions with the increasing DNA content. Now, Zegerman and colleagues show that four factors that control the rate of DNA replication initiation at high $\mathrm{N} / \mathrm{C}$ ratios determine the onset of MBT events in Xenopus. Replication initiation requires both cyclin-dependent kinase (CDK) and Dbf4-dependent kinase (DDK) to activate replisomes in S phase. The authors show that levels of DDK subunit Drf1, CDK substrates Recq4 and Treslin and their interaction partner Cut5 decline during early cell divisions and become limiting for replication of sperm nuclei in Xenopus egg extracts and for replication initiation in fertilized eggs. Co-overexpression of these four factors shortens the cell-cycle length, producing embryos with increased cell numbers and DNA content after MBT, and causes earlier activation of the checkpoint kinase Chk1. This effect is reversible and dose dependent, consistent with a cell-cycle duration determined by replication-factor titration. Transcriptome sequencing of staged embryos reveals that the onset of zygotic gene expression is delayed upon overexpression of these replication factors, suggesting that transcription initiation at MBT is also controlled by cell-cycle length. Embryos overexpressing Drf1, Recq4, Treslin and Cut5 fail to complete gastrulation and die before neurula stage. However, limiting replisome assembly by depleting Cdc6 suppresses lethality, indicating that the developmental defects associated with factor overexpression are the result of changes in replication initiation frequency. These studies suggest that titration of key DNA-replication factors can promote the cell-cycle changes that control MBT events. (Science doi:10.1126/science.1241530, published online 1 August 2013) 